\title{
Estudio de la resistencia a la abrasión de capas vitrocerámicas depositadas sobre acero
}

\author{
J.M. GÓMEZ de SALAZAR, M.I. BARRENA, A. SORIA, M. MENÉNDEZ, A. GONZÁLEZ \\ Dpto. CC. de los Materiales e Ing. Metalúrgica. Facultad de CC. Químicas. Universidad Complutense de Madrid. Madrid.
}

Se han realizado ensayos de desgaste, tanto en placas esmaltadas sin refuerzo como en esmaltes con distinto porcentaje de carga cerámica (10 y 20\% en peso), y se han comparado frente al del acero base pulido. Las medidas de resistencia a la abrasión se han obtenido utilizado un tribómetro con bola de acero al cromo de $6 \mathrm{~mm}$ de diámetro. El aumento de la superficie de contacto bola-placa a medida que aumenta la distancia recorrida, provoca aumentos exponenciales en los desgastes en el caso del acero pulido. Sin embargo, en el caso de las chapas esmaltadas no se observa este comportamiento, debido a la mayor resistencia al desgaste de las mismas. Cuando las capas esmaltadas están reforzadas con cargas cerámicas, la resistencia al desgaste aumenta con el porcentaje de refuerzo cerámico

Palabras clave: esmalte; carga cerámica; resistencia al desgaste; indentación; tenacidad de fractura.

\section{Abrasion resistance of glass-ceramic layers deposited on steel.}

Wear test has been completed on two types of layered materials: a non-reinforced enamel plate and a ceramic reinforced enamel plate. The latter has been tested in two forms: with $10 \mathrm{wt} . \%$ and $20 \mathrm{wt}$. \% ceramic particles reinforcement. For comparison the same test has also been completed on polished steel. Wear resistance was measure using a tribometer, equipped with a chromium steel sphere of $6 \mathrm{~mm}$ diameter. The sphere-plate surface contact area increases with distance covered, which in the polished steel provokes exponential increments in wear values. This exponential behaviour was not observed in the enamel plates due to their greater wear resistance. The reinforcement of the enamel plates with ceramic particles increases the wear resistance. A higher percentage of ceramic reinforcement results in a greater wear resistance of the plates.

Key words: enamels, ceramic particles, wear resistance, indentation, fracture toughness.

\section{INTRODUCCIÓN}

Los esmaltes vitrocerámicos se aplican sobre las superficies proporcionando a estas múltiples cualidades: una capa químicamente inerte, impermeabilidad frente a líquidos y gases, fácil limpieza, buena resistencia mecánica y elevada resistencia al rayado y a la abrasión. Dichas propiedades se verán incrementadas introduciendo cargas cerámicas en el esmalte base (1).

Los esmaltes vitrificables están constituidos principalmente por un material solidificado de naturaleza vítrea inorgánica producido por fusión, y que se conoce con el nombre de frita. El resto de componentes suelen ser arcillas, electrolitos, óxidos colorantes, agua, etc. Estos materiales son fundamentalmente mezclas complejas de:

1.- Óxidos formadores de red vítrea, como el $\mathrm{SiO}_{2}$ y el $\mathrm{B}_{2} \mathrm{O}_{3}$.

2.- Óxidos modificadores. Alcalinos y alcalinotérreos que se introducen con objeto de romper algunos enlaces, generalmente entre el silicio y el oxígeno, consiguiéndose bajas temperaturas de fusión, de trabajo y de reblandecimiento.

3.-Óxidos intermedios, que sustituyen a átomos como el sili-

cio, introduciendo propiedades ópticas y aumentando la capacidad de absorción de determinadas radiaciones (2).

Por lo tanto, para la producción de vidriados y esmaltes, y bajo el punto de vista de evitar defectos, optimizar el producto o desarrollar nuevos esmaltes, se deberá tener en cuenta el control de los fenómenos de inmiscibilidad, nucleación y cris- talización, que son característicos de las composiciones vítreas. Sin embargo, si bien es importante controlar las condiciones físicas y químicas de los esmaltes, también lo es considerar las interacciones de éste con el propio soporte metálico (adherencia), así como la influencia del propio soporte.

Los procesos de fabricación de estos esmaltes determinan su estructura interna y las características de su superficie, parámetros ambos de los que dependen sus propiedades. En todos los casos, las etapas del proceso de fabricación que más influyen sobre las propiedades son los tratamientos térmicos. La temperatura es el factor que regula la cinética de los procesos (3).

Los tratamientos térmicos posteriores (segundas cocciones, recocidos...) podrán introducir modificaciones o ajustes controlados de esa estructura básica pero sin alterarla en lo fundamental. El producto obtenido debe responder a una caracterización lo más precisa posible, expresada a través de normas, métodos de análisis y ensayos, y especificaciones de requisitos a cumplir por el producto.

Hasta ahora, las distintas teorías enunciadas respecto a los mecanismos de reacción en la interfase esmalte/acero durante el ciclo de cocción del esmalte, se basan en simples reacciones directas redox entre el soporte metálico ó sus óxidos y los óxidos de adherencia, los cuales se consideran no alterados por el esmalte (4). 


\section{PROCEDIMIENTO EXPERIMENTAL}

\subsection{Material de partida}

\subsubsection{ACERO SUBSTRATO DE ESMALTACIÓN}

Para la realización de los ensayos de esmaltado, se ha elegido como superficie substrato un acero calidad denominada AP-11 según norma UNE 36093/85 (Tabla I), con una HRB (dureza Rockwell B) de 67 y una resistencia mecánica de 390 $\mathrm{MPa}$, suministrado por Susider S.A. El material se recibió en forma de fleje de 1000×100x13 mm, y fue cortado en piezas cuadradas de 30x30 mm para la realización de los ensayos mecánicos.

\subsection{2.- ESMALTES}

Para el proceso de esmaltado, se han utilizado dos tipos de esmaltes, suministrados por Ferro Enamels Spain: esmalte base denominación RTU-8030 y frita de fundición denominación MFPE-770/S (composiciones patentadas). Como carga cerámica reforzante del esmalte, se ha utilizado zirconia con un $20 \%$ en peso de Itria, $100 \%$ estabilizado, con denominación 202NS, y de tamaño de grano entre $16-40 \mathrm{~mm}$, suministrado por Sulzer Metco.

Se prepararon superficialmente las piezas de acero mediante desbaste, y posterior decapado con $\mathrm{H}_{2} \mathrm{SO}_{4}$ y deposición de níquel mediante procesos electrolíticos, utilizando I = 0.06-2 A y V = 1.11 voltios, con tiempos de inmersión entre 1 y 3 minutos, alcanzándose un rugosidad superficial (Ra) entre $0.4-0.7 \mu \mathrm{m}$.

La preparación del esmalte base, tanto sin carga como cargado con partículas cerámicas (10 y $20 \%$ en peso), se llevó a cabo en medio acuoso. La homogenización de los esmaltes tiene lugar, en todos los casos, mediante agitación mecánica, y la aplicación de estas suspensiones sobre las superficies metálicas se ha realizado de forma manual mediante pintado. La cocción de los mismos se llevó a cabo en un horno de mufla a temperaturas de $800 \pm 25^{\circ} \mathrm{C}$ durante 4 minutos, obteniéndose una capa de esmalte de entre 1.5 y $2 \mathrm{~mm}$ de espesor y con una rugosidad superficial ( $\mathrm{Ra}$ ) de $0.02 \mu \mathrm{m}$ para el caso de los esmaltes sin carga, y de $0.38 \mu \mathrm{m}$ y $0.55 \mu \mathrm{m}$ para los reforzados con un 10 y un $20 \%$ en peso respectivamente.

Para la preparación de las capas vitrocerámicas de esmalte base+fundición, se parte de chapas de acero esmaltadas sin carga cerámica (esmalte base), para posteriormente ser aplicadas capas de frita de fundición, dependiendo de los casos, sin o con carga cerámica, utilizando el mismo procedimiento de aplicación y cocción que en los esmaltes base.

Los ensayos de desgaste se llevaron a cabo en un tribómetro con bola de acero al cromo de $6 \mathrm{~mm}$ de diámetro, utilizando una velocidad angular constante de 100 r.p.m. y una carga aplicada de $500 \mathrm{~g}$. Se realizaron tres medidas experimentales del desgaste a 2, 4 y 6 horas en el caso del acero desnudo, y de 6 horas para las placas vitrocerámicas. Así mismo, fueron calculadas las pérdidas de masa de la bola y la placa, en función del tiempo de ensayo y espacio recorrido por la bola (5).

Se llevaron a cabo cinco ensayos de dureza por condición, empleando un durómetro Vickers Akashi AVK-AII utilizando una carga de $1 \mathrm{~kg}$. Así mismo, se ha utilizado un sistema de captura de imagen FPS 60 y un software de medida con el fin de cuantificar la tenacidad de fractura a partir de las grietas originadas en el proceso de indentación (6).
TABLA I. COMPOSICIÓN EN PORCENTAJE EN PESO DEL ACERO DENOMINADO AP-11.

\begin{tabular}{|c|c|c|c|c|c|c|}
\hline Aleantes & $\mathbf{C}$ & $\mathbf{S i}$ & $\mathbf{M n}$ & $\mathbf{P}$ & $\mathbf{A l}$ & $\mathbf{S}$ \\
\hline $\begin{array}{c}\text { Porcentaje en } \\
\text { peso }\end{array}$ & 0.035 & 0.016 & 0.29 & 0.012 & 0.036 & 0.011 \\
\hline
\end{tabular}
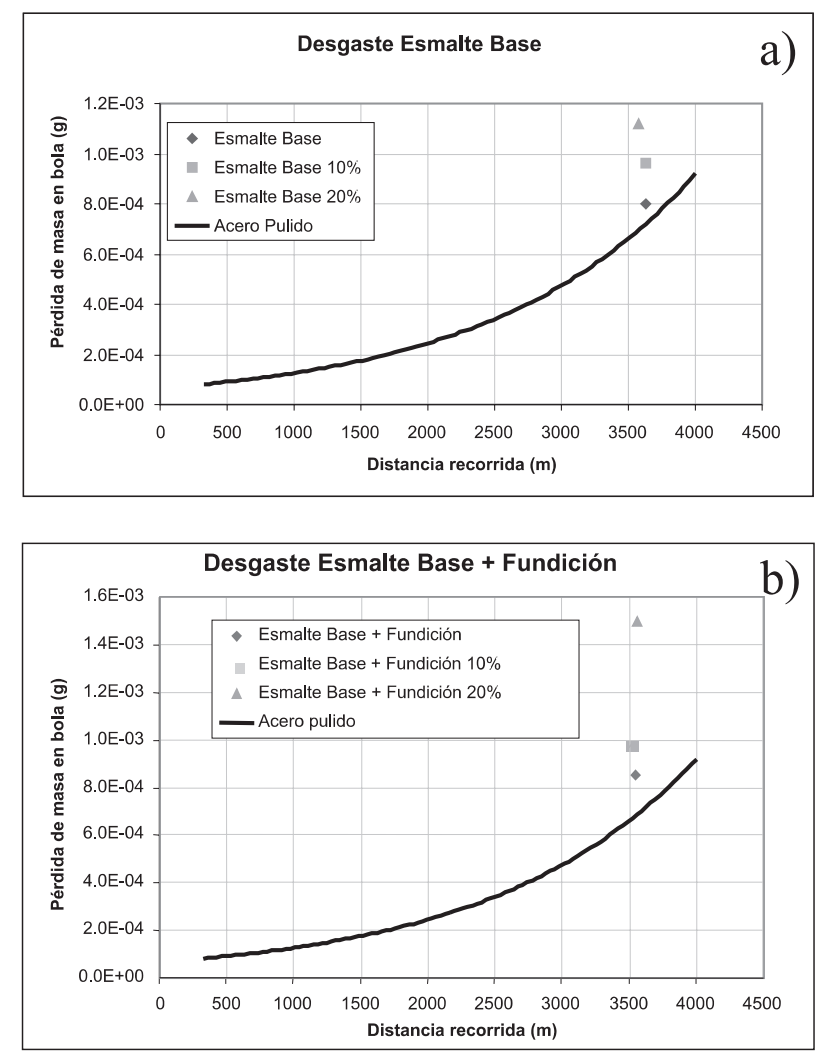

Figura 1. Pérdida de masa en bola vs. distancia recorrida por la bola durante el ensayo de desgaste. a) Esmalte base, $10 \%$ y $20 \%$ en peso de carga cerámica, respecto a la del acero pulido; b) Esmalte base + fundición, $10 \%$ y $20 \%$ en peso de carga cerámica, respecto a la del acero pulido.

\section{RESULTADOS Y DISCUSIÓN}

\subsection{Desgaste}

Los ensayos de desgaste han permitido comparar cualitativamente la resistencia a la abrasión de cada uno de los esmaltes ensayados, así como la de los esmaltes con distinto porcentaje de carga cerámica, frente a la del acero pulido.

La pérdida de masa se podría medir tanto sobre la bola como sobre la placa. En el caso de las medidas sobre la bola, la adición de cargas cerámicas provoca un aumento de la resistencia al desgaste (Fig. 1). Así pues, la huella producida por la bola en el esmalte sin carga cerámica presenta una morfología homogénea, no siendo así en los esmaltes con carga 

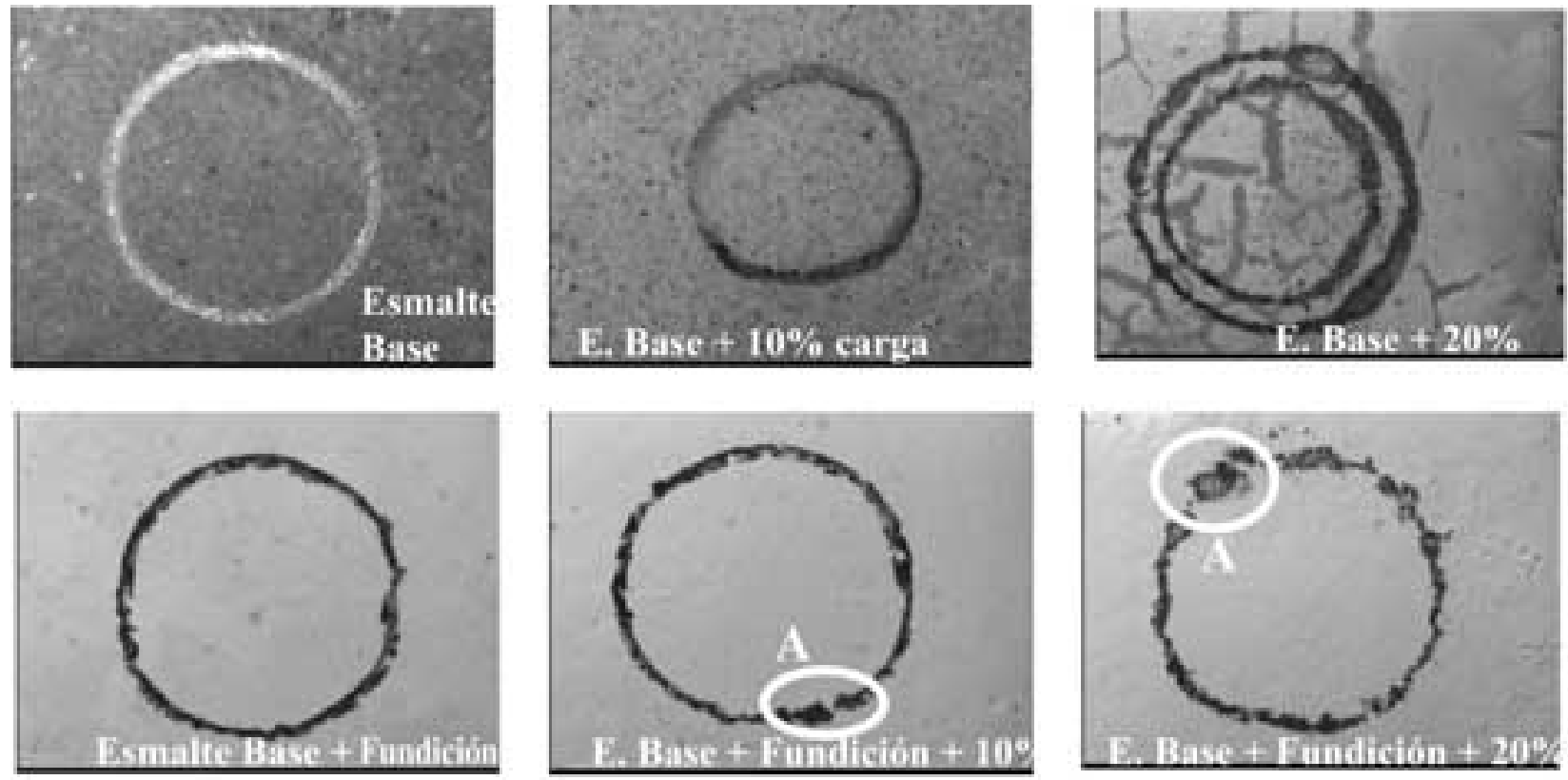

Figura 2. Macrografías de las huellas de desgaste en las superficies vitrocerámicas en función de su naturaleza y el porcentaje de carga cerámica. Las zonas A muestran arranque de material.
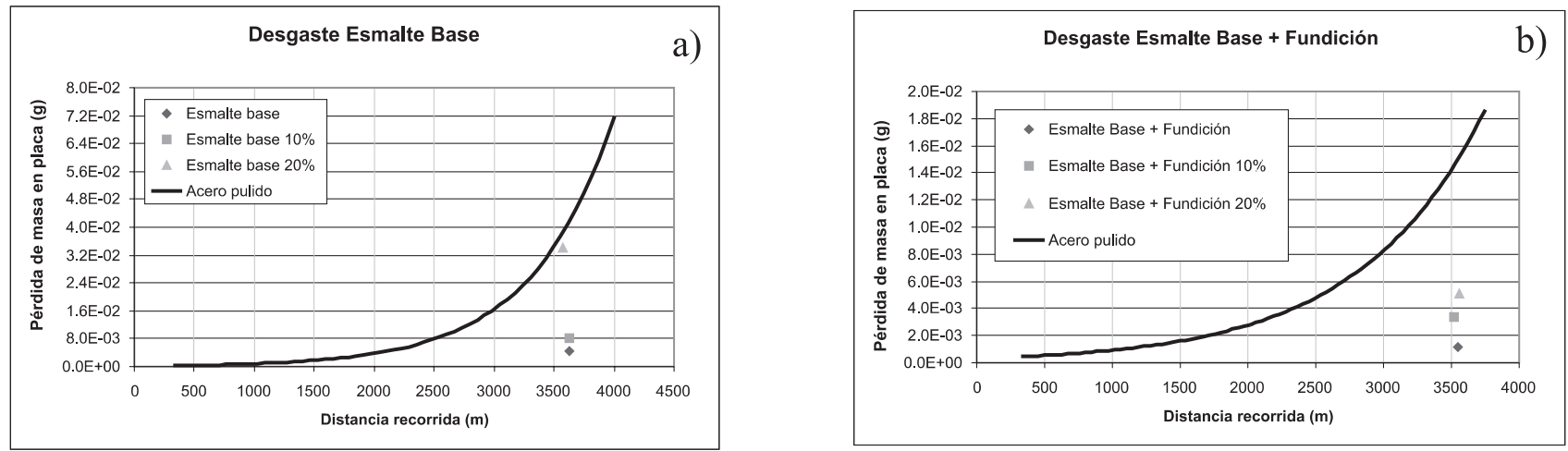

Figura 3. Pérdida de masa en la placa vs. distancia recorrida por la bola durante el ensayo de desgaste. a) Esmalte base, $10 \%$ y $20 \%$ en peso de carga cerámica, y acero pulido; b) Esmalte base + fundición, $10 \%$ y $20 \%$ en peso de carga cerámica y acero pulido.

cerámica (Fig. 2). Las huellas producidas en los esmaltes con mayor carga cerámica son heterogéneas, con zonas en las que se ha arrancado material (Zonas A). La falta de homogeneización de la carga cerámica en el seno del esmalte, ha dado lugar a la existencia de zonas de elevada dureza como consecuencia de un endurecimiento local, que por falta de adherencia han sido arrancadas durante el ensayo, obteniéndose valores de perdida de masa sobre la placa que no se ajustan a los valores reales de desgaste sino al desprendimiento de masa vitrocerámica (Figura 3).

\section{2.- Indentación}

Debido al tamaño de las partículas cerámicas y a su falta de homogeneización en el seno del esmalte se obtiene una dispersión de los datos de dureza Vickers HV 1, por lo que no se han considerado los datos de dureza obtenidos para una posible determinación del grado de endurecimiento por la adición de las cargas cerámicas.

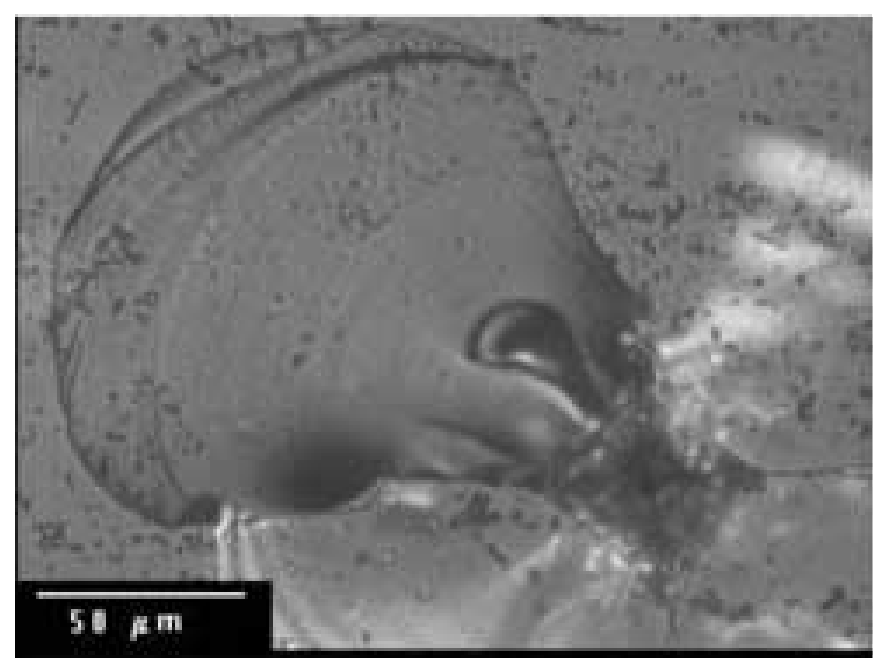

Figura 4. Arranque del material en la arista de una huella de indentación en un esmalte con carga cerámica. 
No obstante, se ha podido comprobar a escala microscópica como, por efecto del endurecimiento local, se producen agrietamientos laterales en las aristas de la huella producida por indentación (cada vez más pronunciadas según aumentan las cargas cerámicas), y como, en algunos casos, se producen arranques de la superficie cerámica en ellas (Fig. 4).

La posibilidad de cuantificar la tenacidad de los esmaltes se ha llevado a cabo mediante medida del tamaño de la grieta radial en las aristas de las huellas. Para el cálculo de los valores de tenacidad $\left(\mathrm{K}_{\mathrm{IC}}\right)$, se han realizado varias aproximaciones. Se ha utilizado la ecuación ampliada en la que se tienen en cuenta los valores de módulo elástico $(\mathrm{E})$ y dureza $(\mathrm{H})$ de cada componente del esmalte cerámico, tanto del propio esmalte como de las cargas cerámicas (7).

$$
K_{I C}=0.016 \cdot\left(\frac{E}{H}\right)^{1 / 2} \cdot \frac{P}{c^{3 / 2}}
$$

siendo C, la longitud de la grieta y P la carga de indentación.

En el caso de obtener la medida sobre un esmalte con carga cerámica, los valores de tenacidad se han calculado aplicando la Ley de las Proporciones (3):

TABLA II. MÓDULO ELÁSTICO Y DUREZA DE LOS ESMALTES EN FUNCIÓN DE LOS PORCENTAJES EN PESO DE LAS CARGAS CERÁMICAS UTILIZADAS

\begin{tabular}{|c|c|c|c|c|c|}
\hline \multicolumn{3}{|c|}{ Esmalte base } & \multicolumn{3}{c|}{ Esmalte base + Fundición } \\
\hline Carga & $\begin{array}{c}\mathbf{E} \\
(\mathrm{GPa})\end{array}$ & $\begin{array}{c}\mathbf{H} \\
(\mathrm{MPa})\end{array}$ & Carga & $\begin{array}{c}\mathbf{E} \\
(\mathrm{GPa})\end{array}$ & $\begin{array}{c}\mathbf{H} \\
(\mathrm{MPa})\end{array}$ \\
\hline Sin Carga & 91 & 6.6 & Sin carga & 90 & 6.5 \\
\hline $\begin{array}{c}\mathbf{1 0 \%} \text { en } \\
\text { peso }\end{array}$ & 103 & 7.7 & $\begin{array}{c}\mathbf{1 0 \%} \text { en } \\
\text { peso }\end{array}$ & 102 & 7.4 \\
\hline $\begin{array}{c}\mathbf{2 0 \%} \text { en } \\
\text { peso }\end{array}$ & 116 & 8.8 & $\begin{array}{c}\mathbf{2 0 \%} \text { en } \\
\text { peso }\end{array}$ & 115 & 8.3 \\
\hline
\end{tabular}

TABLA III. VALORES MEDIOS DE LONGITUD DE GRIETA DE INDENTACIÓN (C) PARA LOS ESMALTES, EN FUNCIÓN DE LOS PORCENTAJES EN PESO DE LAS CARGAS CERÁMICAS UTILIZADAS.

\begin{tabular}{|c|c|c|c|}
\hline \multicolumn{2}{|c|}{ Esmalte Base } & \multicolumn{2}{c|}{ Esmalte base +Fundición } \\
\hline Carga & c (mm) & Carga & c (mm) \\
\hline Sin carga & $0.0747 \pm 0.08$ & Sin carga & $0.0728 \pm 0.08$ \\
\hline $\begin{array}{c}\mathbf{1 0 \%} \text { en } \\
\text { peso }\end{array}$ & $0.0719 \pm 0.08$ & $\begin{array}{c}\mathbf{1 0 \%} \text { en } \\
\text { peso }\end{array}$ & $0.0517 \pm 0.08$ \\
\hline $\begin{array}{c}\mathbf{2 0 \%} \text { en } \\
\text { peso }\end{array}$ & $0.0681 \pm 0.08$ & $\begin{array}{c}\mathbf{2 0 \%} \text { en } \\
\text { peso }\end{array}$ & $0.0392 \pm 0.08$ \\
\hline
\end{tabular}

TABLA IV. VALORES DE TENACIDAD DE FRACTURA (KIC) PARA LOS ESMALTES EN FUNCIÓN DEL PORCENTAJE EN PESO DE LAS CARGAS CERÁMICAS UTILIZADAS

\begin{tabular}{|c|c|c|c|}
\hline \multicolumn{2}{|c|}{ Esmalte Base } & \multicolumn{2}{c|}{ Esmalte base + Fundición } \\
\hline Carga & $\begin{array}{c}\mathbf{K}_{\mathbf{I C}}(\mathbf{M P a} \\
\left.\mathbf{m}^{\mathbf{1 / 2}}\right)\end{array}$ & Carga & $\begin{array}{c}\mathbf{K}_{\mathbf{I C}}(\mathbf{M P a} \\
\left.\mathbf{m}^{\mathbf{1 / 2}}\right)\end{array}$ \\
\hline Sin carga & 0.3 & Sin carga & 0.3 \\
\hline $\begin{array}{c}\mathbf{1 0 \%} \text { en } \\
\text { peso }\end{array}$ & 0.9 & $\begin{array}{c}\mathbf{1 0 \%} \text { en } \\
\text { peso }\end{array}$ & 1.5 \\
\hline $\begin{array}{c}\mathbf{2 0 \%} \text { en } \\
\text { peso }\end{array}$ & 1.0 & $\begin{array}{c}\mathbf{2 0 \%} \text { en } \\
\text { peso }\end{array}$ & 2.3 \\
\hline
\end{tabular}

Para el caso de los esmaltes, los valores de módulo elástico y dureza, al no estar tabulados en la bibliografía, se han utilizado valores correspondientes a un vidrio silicoaluminoso de composición similar al utilizado en este trabajo (8).

Los valores de módulo elástico y dureza de las cargas cerámicas están tabulados en la bibliografía (9), y han sido recalculados para cada porcentaje de carga cerámica (Tabla II).

De las huellas producidas por indentación, se han seleccionado para el estudio de la longitud de la grieta aquellas que presentaban una morfología nítida de grieta en ángulo, rotura típica de este tipo de materiales (Fig. 5). Se ha evitado estudiar huellas que presentaran defectos en la proximidad de las mismas (Fig. 6) que podrían llevar a error por estar situadas en lugares de acumulación de tensiones residuales provocada por los diferentes coeficientes de expansión térmica del esmalte $\left(1-1.2 \times 10^{-6} \mathrm{~K}^{-1}\right)$ y el óxido empleado como carga cerámica $\left(8.0 \times 10^{-6} \mathrm{~K}^{-1}\right)$.

La tabla III recoge los valores de longitud de grieta de indentación medidos para cada una de las probetas.

Una vez conocidos todos los parámetros, se calculan los valores de Tenacidad de Fractura $\left(\mathrm{K}_{\mathrm{IC}}\right)$ para cada esmalte $\mathrm{y}$ porcentaje de carga cerámica (Tabla IV). Se comprueba no

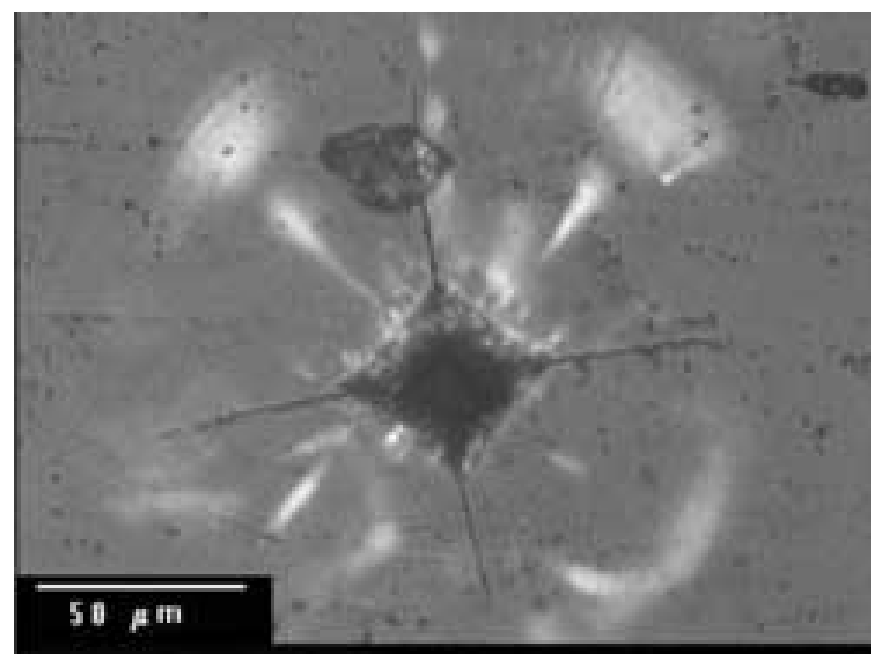

Figura 5. Morfología de grieta en ángulo respecto a una huella de indentación.

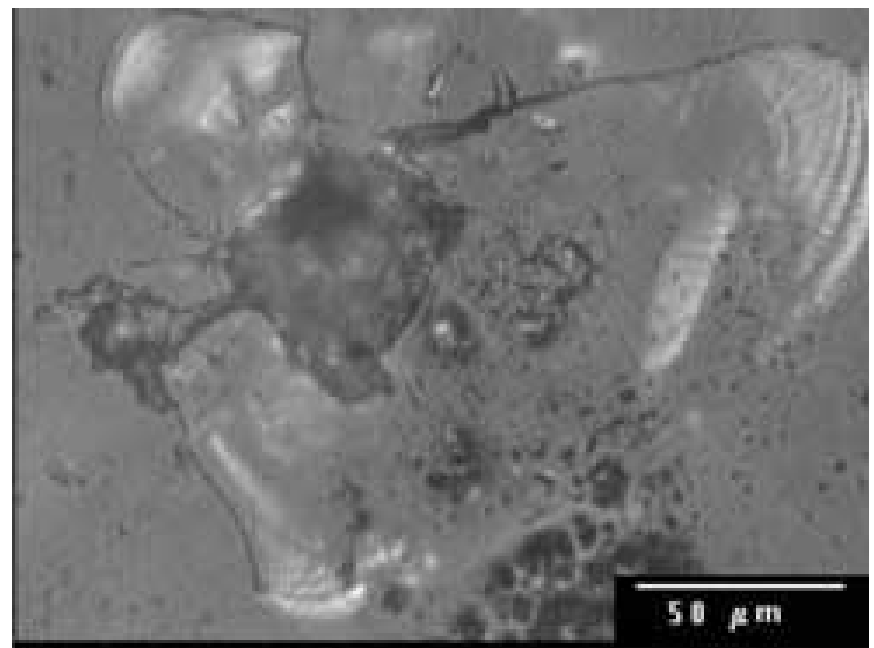

Figura 6. Micrografía de la huella de dureza que presenta defectos en sus proximidades. 
obstante que, como era de esperar, los valores de Tenacidad de Fractura $\left(\mathrm{K}_{\mathrm{IC}}\right)$ aumentan a medida se aumenta el porcentaje de carga cerámica en el esmalte. La presencia de partículas dispersas en el seno del esmalte provoca el anclaje de la grieta nucleada, impidiendo su avance. Por lo tanto, mayor cantidad de partículas de refuerzo dan lugar a mayores puntos de anclaje de grieta, disminuyendo así su longitud y consecuentemente aumentando el valor de Tenacidad de Fractura.

Por otro lado comparando los datos obtenidos de Tenacidad de Fractura, en las mismas condiciones de carga, para el esmalte base son menores que para el esmalte base + fundición. Si tenemos en cuenta que la incorporación de circona en el vitrocerámico tiene lugar a través de la formación de cicón $\left(\mathrm{ZrSiO}_{4}\right)$ en la interfase (10), el mayor o menor grado de formación de este compuesto será pues, el que determine el mecanismo de propagación de la grieta entre la matriz y el refuerzo. Por lo tanto, el hecho de que el esmalte base presente menor valores de tenacidad que el esmalte base + fundición, puede ser debido a las reacciones interfaciales que tienen lugar durante el proceso de cocción. Estos productos de reacción interfacial están siendo objeto de estudio mediante técnicas de TEM y EDX.

\section{CONCLUSIONES}

Como era de esperar la Resistencia a la Abrasión de las capas vitrocerámicas es más elevada que la del metal desnudo y, así mismo, esta propiedad se incrementa con la adición de cargas cerámicas. Cuando el porcentaje en peso de carga cerámica es elevado ( $20 \%$ en peso) se producen zonas de endurecimiento local por acumulación de tensiones residuales, en las que la dureza de la capa vitrocerámica es elevada, provocando el desprendimiento superficial de la misma.

El ensayo de indentación ha revelado que el valor de Tenacidad de Fractura $\left(\mathrm{K}_{\mathrm{IC}}\right)$ de la capa vitrocerámica se ve aumentado con el incremento de carga cerámica.

Se ha comprobado que el esmalte base+fundición con carga cerámica, muestra mayor tenacidad de fractura que el esmalte base con igual contenido de cerámico.

\section{AGRADECIMIENTOS}

Agradecimiento al Proyecto: CAM 07N/0038/98 y a la empresa Ferro Enamel Spain.

\section{BIBLIOGRAFIA}

1. E.A. Mari, "Los Materiales Cerámicos", Librería y Editorial Alsina, Buenos Aires, (1998).

2. J.M. Rincón, J. Cardá y J. Alarcón, “Nuevos productos y tecnologías de esmaltes y pigmentos cerámicos", Editorial Faenza Editrice Ibérica SL, Castellón, (1992).

3. A.I. Andrews, "Porcelain Enamels", Second Edition, The Garrad Press Publishers, Champaign, Illinois, (1961).

4. R.A. Eppler, "Glasses science and technology", Vol I, Editorial D.R. Uhlmann and N.J. Kridl, Academic Press, New York, 301-338, (1983).

5. Norma ASTM G99-90: "Standard Test method for Wear testing with a Pinon-Disk Apparatus", ASTM Standards, (1990).

6. Norma UNE ENV 843-4: “Cerámicas Técnicas Avanzadas. Cerámicas Monolíticas. Propiedades mecánicas a Temperatura ambiente. Parte 4: Ensayo de dureza Vickers, Knoop y Rockwell superficial”, AENOR, (1995).

7. G.R. Anstis, P. Chantikul, B.R. Lawn and D.B. Marshall, "A critical evaluation of indentation techniques for measuring fracture toughness: I, Direct crack measurements", J. Am. Ceram. Soc., 64, 533-538, (1981).

8.R.F. Cook, G.M. Pharr, "Direct Observation and Analysis of Indentation Cracking in Glasses and Ceramics", J. Am. Ceram. Soc., 73 (4), 787-817, (1990).

9. "Surface cleaning, finishing and coating. Ceramic Coating", Metals Handbook, Ninth Edition, Vol. 5, Edit. AMS, Metal Park, Ohio, USA, (1982).

10. "Refuerzo de materiales vítreos para su uso en aplicaciones tradicionales" Tesis Doctoral de Dña. Esther Ocaña García (Instituto de Cerámica y Vidrio - CSIC) presentada en la Universidad Autónoma de Madrid, Madrid (2000). 